\title{
A Simple X-Ray Emitter
}

\author{
Hiroaki MuraKami,* Ryoichi Ono,** Atsuhiko HiraI,** Yoshinori HosoKawa,** and Jun KawaI* \\ *Department of Materials Science and Engineering, Kyoto University, Sakyo-ku, Kyoto 606-8501, Japan \\ **X-Ray Precision, Inc., 2 Miyanohigashimachi, Kisshoin, Minami-ku, Kyoto 601-8510, Japan
}

\begin{abstract}
A compact X-ray emission instrument is made, and the X-ray spectra are measured by changing the applied electric potential. Strong soft X-rays are observed when evacuating roughly and applying a high voltage to an insulator settled in this device. The X-ray intensity is higher as the applied voltage is increased. A light-emitting phenomenon is observed when this device emits X-rays. The present X-ray emitter is made of a small cylinder with a radius of $20 \mathrm{~mm}$ and a height of $50 \mathrm{~mm}$. This X-ray generator has a potential to be used as an X-ray source in an X-ray fluorescence spectrometer.
\end{abstract}

(Received February 12, 2005; Accepted May 17, 2005)

\section{Introduction}

Energy-dispersive X-ray fluorescence (EDXRF) spectrometry is a well-established technique with high sensitivity for a wide range of elements. Recently, portable or handy EDXRF spectrometers have been developed for art, ${ }^{1-4}$ archaeological, ${ }^{5-7}$ environmental, ${ }^{8-10}$ and forensic analyses. Radioisotopes, small air-cooled X-ray tubes, or pyroelectric X-ray emitters are used. ${ }^{11-19}$

Terasawa found that the charge-up of an insulator produced $\mathrm{X}$-rays. ${ }^{20} \mathrm{He}$ invented an X-ray device, as shown in Fig. $1,{ }^{21}$ where applying a high voltage on an insulator produced strong $\mathrm{X}$-rays. Then, Kawai et al.22 found that a vacuum of between 0.03 and 0.04 Torr was the best for X-ray emission. Brownridge $^{23}$ earlier proposed a pyroelectric insulator to produce X-rays, and many interesting phenomena were found for pyroelectric crystals. ${ }^{24}$

In the present work, the $\mathrm{X}$-ray generator proposed by Terasawa $^{20}$ was made and an independent verification of Terasawa is conducted. We measured X-ray spectra from the $\mathrm{X}$-ray emitter and observed visible-light emission. Its correlation with the X-ray intensity was considered.

\section{Experimental}

A compact cylinder-type vacuum tube, a schematic illustration of which is shown in Fig. 2, with $20 \mathrm{~mm}$ radius and $50 \mathrm{~mm}$ height was made. A photo is shown in Fig. 3, left. The cylinder body was polypropylene, made of a medical disposal syringe. The top and bottom were made of brass. Teflon as an insulator was fixed between the needle-shaped copper electrode and a plate electrode with a spring. The cylinder was roughly evacuated (about $6 \mathrm{~Pa}=0.04$ Torr) by a rotary pump and a high voltage was applied from 1500 to $2900 \mathrm{~V}$ by a DC power supply. The bottom brass was grounded, and to a feedthrough electrode was applied a minus high voltage. In the present paper we assign a high voltage, such as $1500 \mathrm{~V}$, but this means that the feedthrough was applied $-1500 \mathrm{~V}$, and the bottom brass was grounded. We also tried that a high voltage was applied between the top brass and the feedthrough; this way of producing an electric field also produced high intensity X-rays. The vacuum of the cylinder was adjusted by a valve between the rotary pump and the X-ray cylinder, to be $6 \mathrm{~Pa}$, which was suitable for the strongest X-ray emission, according to Ref. 22. The X-rays were emitted through a $150 \mu \mathrm{m}$ thick beryllium window. The profiles of the measured X-ray spectra with the applied voltages of $1800 \mathrm{~V}, 2000 \mathrm{~V}$ and $2400 \mathrm{~V}$ were as follows: (1) The peak positions were $1.71,1.79$, and $1.86 \mathrm{keV}$, respectively. (2) The higher energy side edges of the spectra were $1.97,2.14$, and $2.54 \mathrm{keV}$, respectively. Above these energies, the spectra became zero counts. The spectra were weak, but similar to those measured by a glass cylinder X-ray tube described below.

The X-ray spectra shifted to higher energy as the applied voltage became higher. During X-ray emission, a moving visible light emitting phenomenon was observed inside the cylinder, as shown in Fig. 4, where three representative patterns of light emission are pictured. The light became stronger as the applied voltage became higher. As the operation time became longer, the solder used in the vacuum was melted and sputtered due to the heat originated from the plasma process in the cylinder; consequently, an excess discharge arose along the inner wall, which was covered by solder sputtered, and finally the X-ray emission stopped. The main reason for this short lifetime of the tube was due to the leakage of the vacuum because of the flexible syringe.

To improve this short lifetime, the syringe pipe was replaced

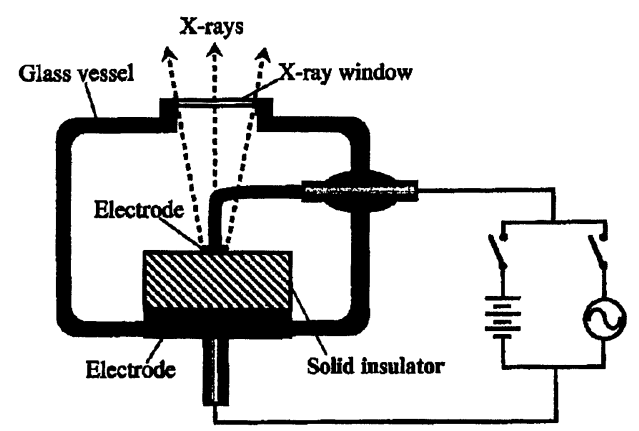

Fig. 1 X-ray emission device proposed by Terasawa. ${ }^{21}$ 
by a glass pipe, as shown in Fig. 3, right. The outer electrode was copper; tungsten wire was used for a feedthrough of the glass. Tungsten was suitable for an airtight feedthrough. The needle-shaped electrode was nickel, because it was easy to bend with elasticity.

A Si-PIN detector, XR-100CR (Amptek, Bedford, MA, USA), with a $7 \mathrm{~mm}^{2}$ effective detection area and an energy resolution of $200 \mathrm{eV}$ for $5.9 \mathrm{keV}$ X-rays was used for measurements. The detector window was $25 \mu \mathrm{m}$ thick beryllium. The detector was thermoelectrically cooled. All of the measurements were performed under the atmospheric pressure for $100 \mathrm{~s}$. The signal from the detector was transfered to a personal computer through an Amptek MCA8000A multi-channel analyzer. The distance between the detector and the generator windows was typically $3.0 \mathrm{~cm}$.

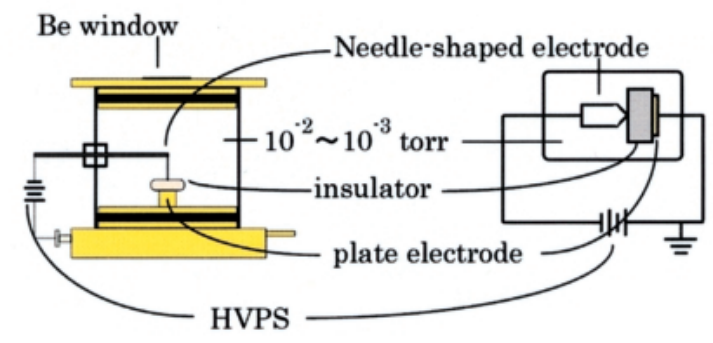

Fig. 2 Schematic illustration of the generator.

\section{Results and Discussion}

The X-ray spectra of the glass cylinder type, the applied voltages of which were 1500, 2000, 2500, and $2900 \mathrm{~V}$, are shown in Figs. $5 \mathrm{a}-\mathrm{d}$. For the spectra a - c, the X-ray intensity became stronger with the increase of the applied voltage. However, spectrum d was weaker than c, as can be seen from Fig. 5. Live time values of the measurements are also shown in Fig. 5; it was $67 \mathrm{~s}$ for c, but $29 \mathrm{~s}$ for d. These values are significantly shorter than Fig. 5a (100 s) and b (97 s). The Xray intensity was too strong in Fig. $5 d$, such that the detector was saturated. Therefore, the distance was increased up to 5.0 $\mathrm{cm}$, and the X-ray spectra at 2500 and $2900 \mathrm{~V}$ were again measured, which are spectra e and $\mathrm{f}$ in Fig. 5. The live time became longer, 99 and 93 s, respectively, and saturation was avoided.

The peak position was $1.29 \mathrm{keV}$ for a, $1.66 \mathrm{keV}$ for $\mathrm{b}, 1.94$ $\mathrm{keV}$ for $\mathrm{c}$ and $\mathrm{e}$, and $2.05 \mathrm{keV}$ for $\mathrm{d}$ and $\mathrm{f}$. The higher energy side edges were 1.43 (a), 2.01 (b), 2.20 (e), $2.60 \mathrm{keV}$ (f). The $\mathrm{X}$-ray spectra shifted to a higher energy as the applied voltage became higher. Visible light was emitted during the emission of X-rays, as shown in Fig. 4.

According to an old X-ray text book, ${ }^{25}$ the present kind of Xray tube was called "gas ion tube" about 100 years ago. In ordinary air, a very few numbers of positive and negative charged ions exist. These charged particles are accelerated by the high electric potential, and bombard the electrodes, which
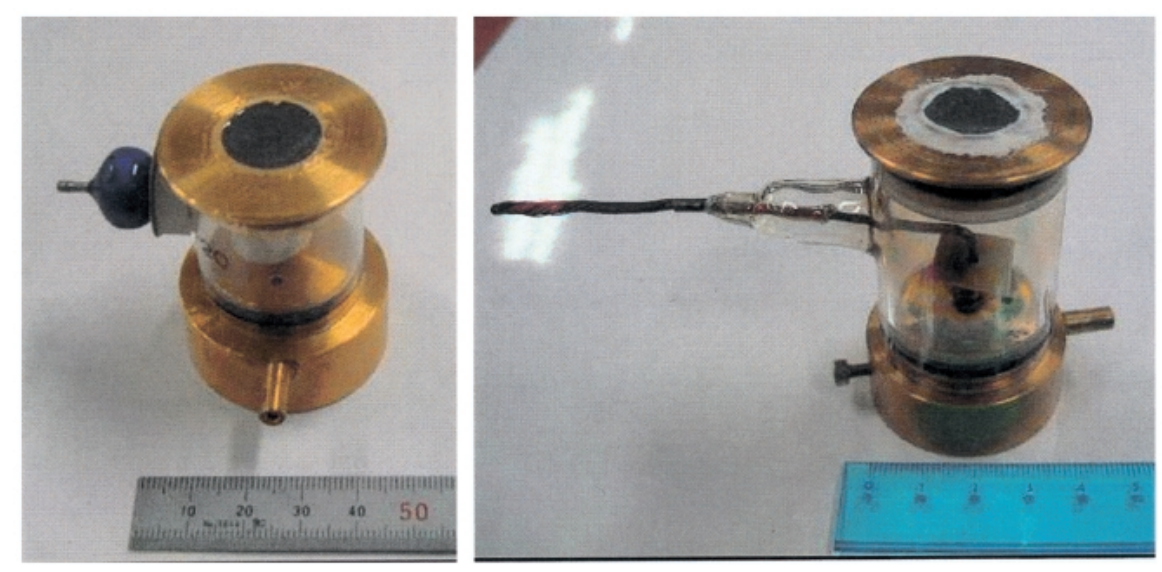

Fig. 3 Photo of the first polypropylene instrument (left) and the second glass instrument (right).
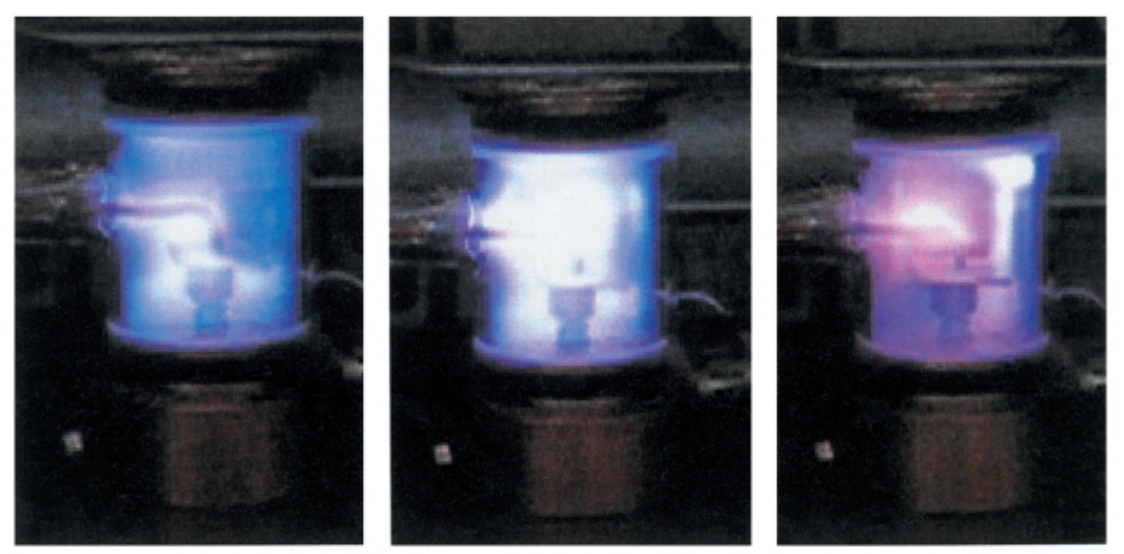

Fig. 4 Photo of the stable light-emitting phenomena. 

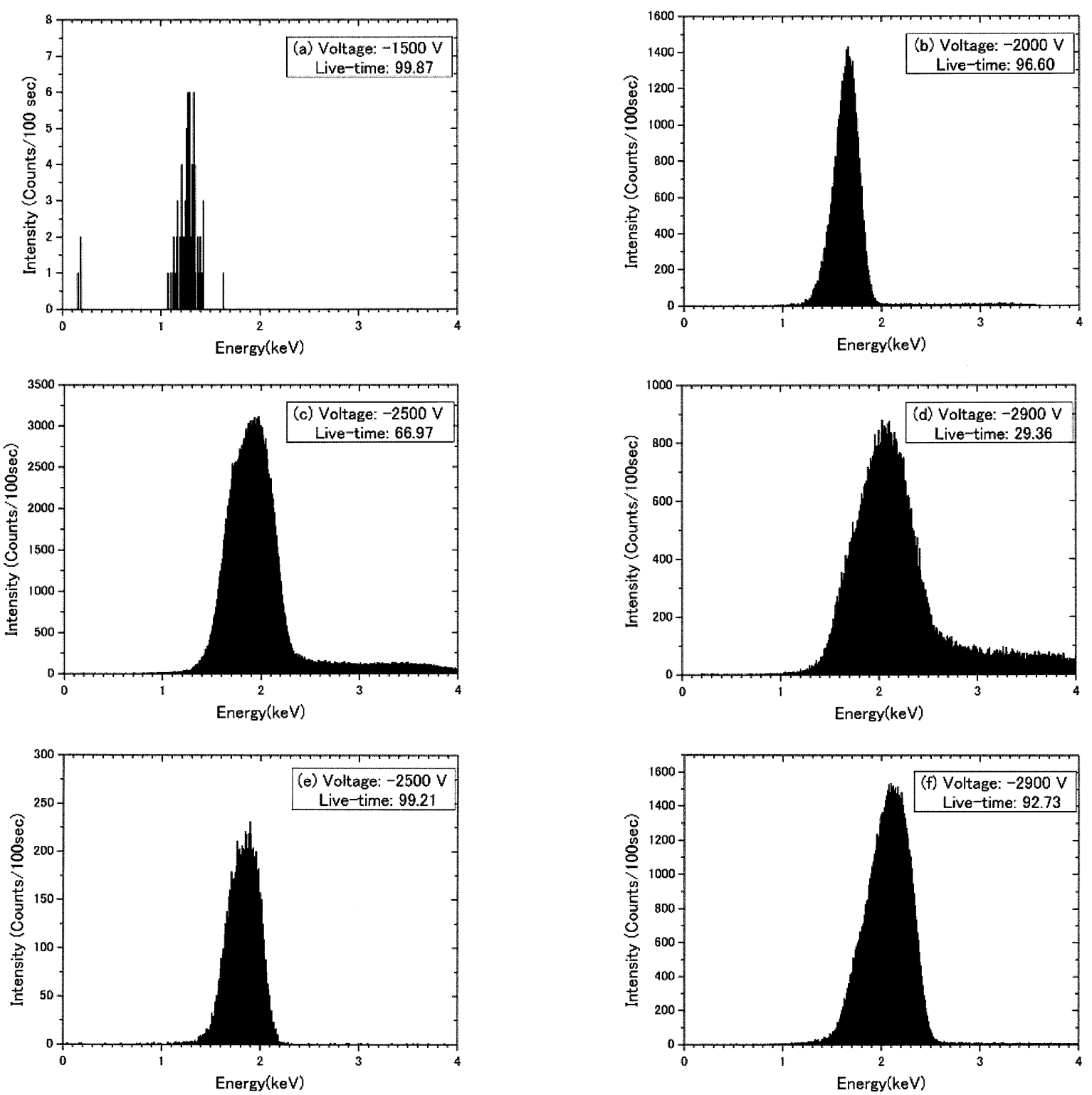

Fig. 5 X-ray spectra of the second instrument. The applied voltage was (a) $-1500 \mathrm{~V}$, (b) $-2000 \mathrm{~V}$, (c) $-2500 \mathrm{~V}$, (d) $-2900 \mathrm{~V}$ (the distance was $3 \mathrm{~cm}$ from generator), (e) $-2500 \mathrm{~V}$, (f) $-2900 \mathrm{~V}$ (the distance was $5 \mathrm{~cm}$ from generator).

then produce another charged particles such as electrons. Therefore, the low pressure is essential for this type of X-ray tubes. It was said that $10^{-2}$ to $10^{-3} \mathrm{~mm} \mathrm{Hg}$ was required. This type of X-ray tubes was quite unstable at an early date. Some were long life and emitted strong X-rays; others were short life and emitted weak X-rays. This inhomogeneity was due to poor control of the vacuum. To improve this inhomogeneity of quality, Coolodge ${ }^{26}$ invented a high-vacuum thermal electron $\mathrm{X}$ ray tube using a filament in 1913. After this year, the "gas ion tube" was forgotten. ${ }^{27}$ However, the Amptek COOL-X is a revival of the gas ion tube, and the present Terasawa type X-ray tube too. We can control the pressure or vacuum quite easily by using a vacuum pump (present experiment) or molecular sieve (COOL-X). The only difference between the Terasawa type and the COOL-X is the way of applying a high voltage, by an electric circuit or by an pyroelectric crystal. Therefore the present Terasawa type X-ray tube has a potential to be used as a similar way as the COOL-X.

\section{Conclusions}

Terasawa type X-ray tubes were constructed, and used to prove the emission of strong X-rays. Low-pressure gas was effective for the X-ray emission. The cylinder dimensions were radius 20 $\mathrm{mm}$ and height $50 \mathrm{~mm}$, and it has a potential to be more compact and smaller dimensions. The present X-ray cylinder could not generate higher energy $\mathrm{X}$-rays than a few $\mathrm{keV}$, because we did not have such an electric power supply. However it would be possible if $10 \mathrm{kV}$ DC power supply is used.

\section{References}

1. A. Castellano and R. Cesareo, Nucl. Instrum. Methods Phys. Res., 1997, B129, 281.

2. R. Cesareo, A. Castellano, G. Buccolieri, and M. Marabelli, 
Nucl. Instrum. Methods Phys. Res., 1999, B155, 326.

3. J. L. Ferrero, C. Roldán, D. Juanes, E. Rollano, and C. Morera, X-ray Spectrom., 2002, 31, 441.

4. J. L. Ferrero, C. Roldán, D. Juanes, J. Carballo, J. Pereira, M. Ardid, J. L. Lluch, and R. Vives, Nucl. Instrum. Methods Phys. Res., 2004, B213, 729.

5. R. Cesareo, G. E. Gigante, P. Canegallo, A. Castellano, J. S. Iwanczyk, and A. Dabrowski, Nucl. Instrum. Methods Phys. Res., 1996, A380, 440.

6. A. Longoni, C. Fiorini, P. Leutenegger, S. Sciuti, G. Fronterotta, L. Strüder, and P. Lechner, Nucl. Instrum. Methods Phys. Res., 1998, A409, 407.

7. G. E. Gigante and R. Cesareo, Radiat. Phys. Chem., 1998, 51,689 .

8. M. B. Bernick and P. R. Campagna, J. Hazard. Mater. 1995, 43, 91

9. M. B. Bernick, D. J. Kalnicky, G. Prince, and R. Singhvi, J. Hazard. Mater., 1995, 43, 101.

10. D. A. Sterling, R. D. Lewis, D. A. Luke, and B. N. Shadel, Environ. Res., 2000, 83, 174.

11. J. Kawai, T. Yamada, and H. Fujimura, Bunseki Kagaku, 2004, 53, 183.

12. H. Ida and J. Kawai, Adv. X-Ray Chem. Anal. Jpn., 2004,
35,81 .

13. H. Ida and J. Kawai, Bunseki Kagaku, 2004, 53, 753.

14. H. Ida and J. Kawai, Anal. Bioanal. Chem., 2004, 379, 735.

15. H. Ida and J. Kawai, Anal. Sci., 2004, 20, 1211.

16. H. Ida and J. Kawai, J. Anal. At. Spectrom., 2004, 19, 1524.

17. H. Ida and J. Kawai, Spectrochim. Acta, Part B, 2004, 60 , 89.

18. H. Ishii, N. Sato, M. Kawano, T. Mesaki, and J. Kawai, Bunseki Kagaku, 2005, 54, 321.

19. H. Ishii, H. Miyauchi, T. Hioki, and J. Kawai, $A d v . X$-Ray Chem. Anal. Jpn., 2005, 36, 225.

20. M. Terasawa, J. Phys. Soc. Jpn., 1968, 25, 1199.

21. M. Terasawa, Japanese Patent, 1974, Showa 49-23995.

22. J. Kawai, N. Inada, and K. Maeda, Adv. X-Ray Chem. Anal. Jpn., 1998, 29, 203.

23. J. D. Brownridge, Nature, 1992, 358, 287.

24. J. A. Geuther and Y. Danon, J. Appl. Phys., 2005, 97, 074109.

25. U. Yoshida and K. Tanaka, "X-sen Kesshougaku", 1943, Sanseido, Tokyo, 1.

26. W. D. Coolidge, Phys. Rev., 2nd ser., 1913, 2, 409.

27. G. W. C. Kaye, "X Rays", 1923, Longmans, London, 43. 